Supporting Information

\title{
Freezable and Unfreezable Hydration Water: Distinct Contributions to Protein Dynamics Revealed by Neutron \\ Scattering
}

Naoki Yamamoto ${ }^{1, *}$, Maiko Kofu², Kenji Nakajima ${ }^{2}$, Hiroshi Nakagawa ${ }^{2,3}$, Naoya

Shibayama ${ }^{1}$

${ }^{1}$ Division of Biophysics, Department of Physiology, Jichi Medical University, 3311-1

Yakushiji, Shimotsuke, Tochigi 329-0498, Japan

${ }^{2} \mathrm{~J}-\mathrm{PARC}$ Center, Japan Atomic Energy Agency, 2-4 Shirakata, Tokai-mura, Naka-gun,

Ibaraki 319-1195, Japan

${ }^{3}$ Materials Sciences Research Center, Japan Atomic Energy Agency, 2-4 Shirakata,

Tokai-mura, Naka-gun, Ibaraki 319-1195, Japan

Corresponding Author

*E-mail: nyamamoto@jichi.ac.jp 
Sample preparation and the detail of the neutron scattering experiment

We used hen egg lysozyme with a controlled amount of hydration as a sample for neutron experiments. To exchange solvent $\mathrm{H}_{2} \mathrm{O}$ or exchangeable proton of protein by $\mathrm{D}_{2} \mathrm{O}$ or deuterium, the protein powder was dissolved in $\mathrm{D}_{2} \mathrm{O}$, incubated for $12 \mathrm{~h}$, followed by lyophilization. The procedure was repeated for three times. This lyophilized sample was defined as the dehydrated state. Then the dehydrated powder was hydrated by $\mathrm{D}_{2} \mathrm{O}$.

In neutron scattering, the energy resolution (full width at half maximum) was $0.1 \mathrm{meV}$, which corresponds to the resolution time of $\sim 2 \times 10^{-11} \mathrm{~s}$. This means that the dynamics faster than $2 \times 10^{-11} \mathrm{~s}$ can be detected at this setup. The obtained $q$ range was $0.1 \leq q \leq$ $2.5 \AA^{-1}$ where the resolution, $\Delta q / q$, was $\sim 1.1 \%$. The sample temperature was raised at the rate of $1.0-1.5 \mathrm{~K} / \mathrm{min}$ or decreased at the rate of $0.25 \mathrm{~K} / \mathrm{min}$ without over-heating or over-cooling. After a desired temperature was achieved, the sample was held at least for $10 \mathrm{~min}$ before measurement. Precise control of the sample temperature is crucial for elucidating temperature hysteresis. It was confirmed that the sample temperature was uniformly held within $\pm 0.5 \mathrm{~K}$ using two thermometers that were attached on the top and bottom of the sample cell. 